Cita: Carvalho, R; Monteiro, D; Rodrigues, F (2022). Relação entre os estados de humor e a síndrome de Overtraining em jovens atletas. Cuadernos de Psicología del Deporte, 22(1), 279-291

\title{
Relação entre os estados de humor e a síndrome de Overtraining em jovens atletas
}

\section{Relación entre estados de ánimo y síndrome de Overtraining en jóvenes deportistas}

\section{Relationship between mood states and Overtraining syndrome in young athletes}

\author{
Carvalho, $\mathrm{R}^{1}$, Monteiro, D ${ }^{2,3}$, Rodrigues, $\mathrm{F}^{2,4}$ \\ ${ }^{1}$ Escola Superior de Desporto de Rio Maior (ESDRM - IPSantarém), Rio Maior, Portugal; \\ ${ }^{2}$ ESECS - Politécnico de Leiria, Leiria, Portugal; \\ ${ }^{3}$ Centro de Investigação em Desporto, Saúde e Desenvolvimento Humano (CIDESD), Vila Real, \\ Portugal; \\ 4 Centro de Investigação em Qualidade de Vida (CIEQV), Santarém, Portugal.
}

\begin{abstract}
RESUMO
O presente estudo analisou a associação entre estados humor e Síndrome de Overtraining (SOT) em jovens atletas e as possíveis diferenças nos estados de humor e na SOT em função do nível competitivo e em função das idades. Métodos: No total, 219 jovens atletas, com idades compreendidas entre os 13 e os 17 anos $(\mathrm{M}=14.81$; DP $=1.35)$, foram considerados para análise deste estudo. Resultados: Os resultados demonstraram que existe uma relação positiva e significativa entre a depressão, o desajuste ao treino, a confusão (negativo) e a tensão com a SOT. Foram, também, encontradas diferenças significativas nos estados de humor hostilidade e depressão em função do nível competitivo e nos estados de humor confusão (positivo) e hostilidade em função da idade. Conclusão Estes dados indicam, não só, que existe uma associação positiva entre estados de humor negativos e a SOT, como a existência de diferenças entre níveis competitivos e entre idades, sendo, por isso, necessário ter em conta essas variáveis.
\end{abstract}

Palavras chave: Estados de Humor; Síndrome de Overtraining; Jovens atletas; Nível competitivo

\section{RESUMEN}

Este estudio analizó la asociación entre los estados de ánimo y el Síndrome de Overtraining (SOT) en deportistas jóvenes y las posibles diferencias en los estados de ánimo y en SOT considerando el nivel competitivo y los grupos de edad. Métodos: En total, 219 atletas jóvenes, con edades entre 13 y 17 años $(\mathrm{M}=14.81$; DT = 1.35), fueron considerados para el análisis en este estudio. Resultados: Los resultados demuestran que existe una relación positiva y significativa entre depresión, inadaptación al entrenamiento, confusión (negativa) y tensión con SOT. También se encontraron diferencias significativas en los estados de ánimo, ira y depresión a nivel competitivo, y en confusión (positiva) e ira según la edad. Conclusiones: Estos datos indican, no solo, que existe una asociación positiva entre estados de ánimo negativos y SOT, así como la existencia de diferencias entre niveles competitivos y entre grupos de edad, por lo que es necesario tener en cuenta estas variables.

Palabras clave: Estados de ánimo; Síndrome de Overtraining; Atletas jóvenes; Nivel competitivo 


\title{
Humor e sobretreinamento em jovens atletas
}

\begin{abstract}
This study analyzed the association between mood states and Overtraining Syndrome (OTS) in young athletes and the possible differences in mood states and in OTS considering the competitive level and age groups. Methods: In total, 219 young athletes, aged between 13 and 17 years old $(\mathrm{M}=14.81 ; \mathrm{SD}=1.35)$, were considered for analysis in this study. Results: The results demonstrate that there is a positive and significant relationship between depression, maladjustment to training, confusion (negative) and tension with OTS. Significant differences were also found in the mood states, anger and depression at the competitive level, and in confusion (positive) and anger according to age. Conclusions: These data indicate, not only, that there is a positive association between negative mood states and OTS, as well as the existence of differences between competitive levels and between age groups, therefore, it is necessary to take these variables into account.
\end{abstract}

Keywords: Mood States; Overtraining Syndrome; Young Athletes; Competitive level

\section{INTRODUÇÃO}

Nos dias de hoje, os atletas são constantemente pressionados a elevar o seu nível desportivo, assim como, a sua performance (Carfagno et al., 2014). Dessa forma, com o aumento do volume de treino, a Síndrome de Overtraining (SOT) é sempre uma possibilidade, principalmente, se o período de descanso, em relação ao volume de treino, não for respeitado (Meeusen et al. 2013). Neste sentido, é expectável que uma grande percentagem de atletas apresente SOT pelo menos uma vez na sua carreira (Grant et al., 2012). Porém, apesar da incidência ser reconhecida, a prevalência da SOT é ainda um aspeto pouco consensual na literatura, o que segundo Grandou et al. (2020), deve-se à fraca definição dos conceitos que definem a SOT de outras condições semelhantes, tais como Overreaching e Burnout, bem como à dificuldade em elaborar um diagnóstico preciso. Especificamente, é necessário entender que a SOT não é uma condição linear (Kreher, \& Schwartz, 2012), ou seja, não existe um diagnóstico definido, sendo que, as suas características podem-se assemelhar a outros sintomas, como por exemplo, o Overreaching (OR).

Enquanto OR é considerado uma condição na qual os períodos de descanso não são respeitados em relação à carga, volume e intensidade de treino resultando numa performance baixa a curto prazo, a SOT é considerada uma condição a longo prazo ao qual a sua recuperação pode demorar meses (Meeusen et al. 2013).

Existem vários aspetos que podem estar relacionados com a SOT, sendo que, algumas evidências indicam que os aspetos psicológicos, nomeadamente estados de humor, têm vindo a estar relacionados com a SOT (Grant et al., 2012). Tanto os estados de humor como a SOT dependem sempre de diferentes variáveis, tais como a idade, sexo, modalidade e nível desportivo, (Nixdorf et al., 2013) sendo que, variáveis como o nível desportivo (Armstrong, 2011) e a idade (Aoki, 2013) têm apresentado alguma influência nesse aspeto. Os estados de humor têm sido considerados como determinantes da SOT (Carter et al., 2014) sendo assim importante analisar os estados de humor e a sua relação com a SOT e a forma como diferem no contexto de diferentes variáveis, como o nível desportivo e a idade.

\section{Conceptualização de Síndrome de Overtraining}

A SOT tem sido definida como uma resposta crónica ao desrespeito pela recuperação em relação ao volume, intensidade e carga do treino causando, dessa mesma forma, uma performance reduzida (Meeusen et al. 2013). Igualmente, pode ser causada por inflamação e efeitos secundários no sistema nervoso central, incluindo depressão, fadiga e mudanças neuro hormonais (Kreher, \& Schwartz, 2012). No entanto, os fatores extrínsecos ao treino também desempenham um papel importante no desenvolvimento da SOT, como as pressões do público aumentadas através dos principais meios de comunicação social, limitadas redes de apoio devido à deslocalização, dinâmica de grupo em desportos de equipa e o potencial de lesões que pode terminar carreiras de forma precoce (Rice et al., 2016).

A SOT é, por vezes, confundida com outras condições de características semelhantes, como OR e Burnout, sendo que, apesar de serem condições diferentes, as três apresentam relações no sentido que, um desenvolvimento avançado de OR pode originar SOT e um desenvolvimento avançado da SOT pode originar Burnout (Brooks, \& Carter, 2013). A diferença entre estas três condições não recai sobre a 


\section{Carvalho et al.}

diferenças de sinais/sintomas, mas sim sobre o desenvolvimento dos mesmos sendo na SOT mais desenvolvidos que no OR e considerando o Burnout como uma condição mais desenvolvida que define o final da SOT. Sendo assim, é importante perceber quais os sinais que caracterizam a SOT (Matos et al., 2011), que podem ser muitas vezes físicos, porém, os sinais psicológicos, tais como os estados de humor, têm sido reportados com maior frequência (Tobar, 2012).

Os estados de humor têm sido avaliados como um meio para monitorizar atletas de forma individual e perceber de que forma o aparecimento da SOT possa estar evidente (Meeusen et al., 2013; HernándezMendo, 2011). É preciso perceber que existe inúmeros fatores psicológicos externos que podem causar alterações de humor, tais como relações com o treinador e família, stresse competitivo, problemas pessoais (Grant et al., 2012), sendo que, essas mesmas alterações de humor, causadas por fatores extrínsecos ao treino, podem influenciar o aparecimento $\mathrm{e}$ desenvolvimento da SOT (Rice et al., 2016).

\section{Estados de humor}

Estados de humor são fenómenos inseridos dentro do tipo afetivos e/ou emocionais que se alteram consoante influências positivas ou negativas (Nettle, \& Bateson, 2012; Hernández-Mendo \& Ramos, 1996; Hernández-Mendo \& Ramos, 1995). De acordo com Viana et al., (2001), os estados de humor mais prevalentes e analisados são: a) Tensão, que é caracterizado por tensão músculo-esquelética e preocupação; b) Depressão, que representa um estado emocional de desânimo e solidão; c) Hostilidade que se caracteriza por cólera e antipatia; d) Fadiga que corresponde a um estado de cansaço e baixa energia; e) Vigor que representa um estado de energia e de vigor físico e psicológico; f) Confusão, que, como o próprio nome indica, caracteriza-se por estado de confusão e baixa lucidez, estado este, que pode ser categorizado por confusão (negativo) e confusão (positivo); g) Desajuste ao Treino que se relaciona do ponto de vista conceptual com a depressão, confusão e fadiga e que apresenta correlações com indicadores de SOT.

Apesar de existirem evidências de uma possível relação entre os estados de humor e a SOT, a literatura mostra-se escassa na especificação da relação positiva ou negativa (Kreher, 2016). Estados de humor como tensão, depressão, hostilidade, fadiga e confusão representam indicadores de estados de humor negativos, enquanto vigor, representa um indicador de estado de humor positivo (Yoshihara et al., 2011; Hernández-Mendo, 2002). Estes mesmos estados de humor negativos têm se mostrado mais evidentes em atletas com SOT ou em atletas que se encontram em risco de SOT; enquanto estados de humor positivos apresentam-se menos evidentes nesses mesmos atletas (Cadegiani, 2020; Cadegiani, \& Kater, 2018; Grant et al., 2012; Roberts \& Lane, 2021; Han et al., 2020). Os estados de humor negativos têm, por isso, sido associados positivamente com a SOT, o mesmo acontece com estados de humor positivos, porém, de forma inversa (Schmikli et al., 2011).

Nível competitivo e faixa etária: possiveis diferenças?

Para além da relação entre estados de humor e SOT, parece existir um efeito do nível competitivo relativamente à SOT e aos estados de humor, sendo que, um nível competitivo mais elevado apresenta uma maior prevalência de SOT ou sintomas relacionados à SOT (Bell et al., 2020). Para estes atletas que competem em níveis mais elevados, como nacional ou internacional, para além de apresentarem maior incidência de SOT comparativamente com atletas em níveis regionais, isto devido à carga de treino exigida em níveis mais elevados (Matos et al., 2011), apresentam valores mais elevados de estados de humor negativos, nomeadamente hostilidade e depressão, fator esse, que se deve à constante pressão psicológica durante competições, ao nível de exigência e expectativas colocadas por treinadores, clubes e imprensa (Andrade et al., 2019) e, ao facto, de estes mesmos atletas possuírem um horário e uma agenda competitiva mais rigorosa, afetando, dessa forma, as horas de sono que têm uma influência direta nos estados de humor (Lastella et al., 2014). Num estudo de O'Connor (2007) foram encontrados valores mais elevados de fadiga, depressão e hostilidade em atletas de alta competição comparativamente com atletas que competem em níveis competitivos mais baixos, inclusive, foi analisado o aparecimento e prevalência de SOT sendo os valores mais elevados também em atletas de níveis competitivos mais altos.

Para além do nível competitivo, a idade também apresenta efeitos na SOT e nos estados de humor, sendo que estes atletas de idades mais elevadas, ou até mesmo adultas, apresentam valores superiores de SOT comparativamente a atletas mais jovens (Armstrong et 


\section{Humor e sobretreinamento em jovens atletas}

al., 2011). É possível perceber que, mesmo em idades jovens, existe uma diferenciação, sendo que atletas com idades entre os 15 e os 19 anos tendem apresentar maior incidência de SOT em comparação com atletas mais novos, isto de acordo com a literatura (Matos et al., 2011). Curiosamente, o mesmo não acontece nos estados de humor, sendo que atletas jovens apresentam maiores níveis de estados de humor negativos comparativamente com atletas adultos (ReynosoSánchez et al., 2021: Oliveira et al., 2021). Até mesmo em atletas jovens existe diferenciações entre idades mais reduzidas e mais elevadas, sendo que, atletas com 16 anos de idade apresentam diferenças significativas nos estados de humor negativos, como tensão, depressão, hostilidade e fadiga, comparativamente a atletas com 19 anos de idade (Aoki et al. (2017). Isto acontece, pois, atletas com idades mais reduzidas apresentam maiores fatores de risco para distúrbios alimentares e preocupações com a imagem corporal, inclusive, situações como lesões graves são considerados eventos traumáticos para os mesmos, tudo fatores que apresentam relação com estados de humor negativos (Rice et al., 2016). Para além disso, a idade cronológica desempenha um papel fundamental na capacidade de lidar (coping) com o stress colocado pelo desporto em jovens atletas, como a pressão de pais e treinadores (Mitic et al., 2020), sendo também um dos motivos pelos quais atletas de idades mais jovens apresentam níveis mais elevados de estados de humor negativos (Aoki et al. 2017).

\section{Presente estudo}

Apesar de existirem evidências preliminares na relação entre os estados de humor e a SOT (Cadegiani, \& Kater, 2018), existe uma fraca especificação de que tipo de estados de humor possuem associação com a SOT, sendo necessário uma análise mais completa e detalhada que suporte as evidências de que os estados de humor podem estar relacionados com a SOT (Ten Haaf et al., 2017). Estudos que tenham analisado a relação entre estados de humor e SOT têm apresentado associações com amostras reduzidas (i.e., 51 participantes) sendo essa uma das principais limitações na literatura (Cadegiani, \& Kater, 2018). Assim, o objetivo principal deste estudo consistiu em analisar a associação entre estados de humor e a SOT em jovens atletas portugueses.

Armstrong et al. (2011) menciona que a literatura relativamente ao estudo da SOT em jovens atletas é escassa, sendo, por tal, necessário mais estudos que avaliem as possíveis diferenças na perceção da SOT e aos estados de humor em relação principalmente em atletas mais jovens (Aoki et al., 2017). Assim, em segundo lugar, analisou-se as possíveis diferenças na perceção da SOT e estados de humor em função do nível competitivo e da faixa etária.

Tomando em consideração a literatura existente, especula-se que estados de humor negativos (i.e. tensão, depressão, hostilidade, fadiga e confusão (negativo)) estejam positivamente e significativamente relacionados com a SOT enquanto estados de humor positivos (i.e., vigor e confusão (positivo)) esteja positivamente e significativamente relacionado com a SOT (Cadegiani, 2020; Schmikli et al., 2011). Especula-se que possa haver diferenças significativas na SOT (Armstrong et al., 2011) e nos estados de humor (Andrade et al., 2019; Aoki et al., 2017) em função do nível desportivo e a idade. O mesmo se sucede relativamente ao nível desportivo (Andrade et al., 2019; Bell et al., 2020). Desse modo, como objetivo secundário, pretendeu-se comparar os níveis de SOT e de estados de humor em função do nível de competitividade (i.e., nacional vs. regional) e em função da idade (i.e., 13-15 anos vs. 16-17 anos) de acordo com vários autores (Aoki et al., 2017; Bell et al., 2020).

\section{MATERIAL E MÉTODOS}

\section{Participantes}

Um estudo transversal com 219 atletas, 203 do sexo masculino e 16 do sexo feminino, dos quais 102 competiam a nível regional e 117 a nível nacional. Os participantes apresentaram idades compreendidas entre os 13 e os 17 anos $(M=14,81$; $D P=1,35)$, uma experiência desportiva entre os 3 a 13 anos $(M=7,82$; $\mathrm{DP}=1,89)$, um volume de treino semanal entre 3 e 10 sessões por semana $(\mathrm{M}=3.47$; $\mathrm{DP}=.93)$, cuja duração variou entre 2 e 18 horas semanais $(\mathrm{M}=6,22$; $\mathrm{DP}=$ $2,02)$. Os atletas praticavam diferentes modalidades nomeadamente: Atletismo $(\mathrm{n}=7)$; Ciclismo $(\mathrm{n}=1)$; Futebol $(\mathrm{n}=190)$; Futsal $(\mathrm{n}=1)$; Ginástica $(\mathrm{n}=12)$; Natação $(\mathrm{n}=7)$; Rugby $(\mathrm{n}=1)$.

Para a correta realização deste trabalho, foram convidados a participar, de forma voluntária atletas de acordo com os seguintes critérios de inclusão: atletas de ambos os sexos; que tenham experiência competitiva a nível regional ou nacional; idades compreendidas entre os 13 e os 17 anos; possuidores 


\section{Carvalho et al.}

do consentimento assinado pelos encarregados de educação ou tutores; e que tenham facultado o próprio consentimento de livre participação neste estudo.

\section{Procedimentos}

Após aprovação por parte da Escola Superior de Deporto de Rio Maior, foram contactados por conveniência clubes desportivos e centros de alto rendimento da zona Vale do Tejo. Durante o contacto com os dirigentes, diretores técnicos e presidentes de associações foi explicado todos os objetivos e metodologias do estudo, bem como solicitado a autorização para a aplicação do estudo. Após a sua autorização, foram contactados os treinadores responsáveis pelos atletas, potenciais participantes deste estudo. Para o consentimento da aplicação de questionários aos atletas, tomando em consideração o critério de inclusão da idade, cartas de consentimento informado e autorização para participação neste estudo foram entregues por aos encarregados de educação ou tutores dos potenciais participantes. Todos os participantes menores considerados neste estudo tiveram o consentimento assinado pelos encarregados e deram o seu próprio consentimento de livre participação, conforme estipulada pela declaração de Helsínquia (2013). Os questionários foram preenchidos de forma autónoma pelos participantes através de um questionário online.

\section{Instrumentos}

Para medir os estados de humor recorreu-se ao Profile of Mood States (POMS), traduzido e validado para a população portuguesa por Viana et al. (2001). Este instrumento mede oito estados de humor, nomeadamente: fadiga (e.g., "cansado"), vigor (e.g., "alegre"), hostilidade (e.g., "irritado"), depressão (e.g., "infeliz"), desajuste ao treino (e.g., "inútil"), confusão (negativo; e.g., "confuso"), tensão (e.g., "ansioso") e confusão (positivo; e.g., "competente"). Este instrumento contém 42 adjetivos que são avaliados através de uma escala de 5 pontos variando entre 0 (nada) e 4 (muitíssimo).

Para quantificar os sintomas da síndrome de Overtraining foi utilizado o Société Francaise de Médecine du Sport (SFMS) validado para português por Fernandes et al. (2008). Este instrumento é capaz de medir o grau de sobrecarga em atletas submetidos a programas de treino intensivo. Este questionário é composto por 51 itens (e.g., "O meu nível de rendimento / a minha forma tem piorado.") que são avaliados através de uma escala de 4 pontos variando entre 0 (nunca) e 3 (sempre). Sendo que não existe literatura que sustente a existência de diversas dimensões da SOT, calculou-se a média aritmética.

\section{Análise Estatística}

Tomando em consideração a teoria do limite central, que pressupõe um mínimo de $\mathrm{n}=50$ para uma distribuição normal, não se recorreu a testes de normalidade. Igualmente não foi necessário a análise de valores omissos, dado o questionário ser em formato online com as respostas de preenchimento obrigatório.

A primeira etapa consistiu na análise descritiva (média, desvio-padrão, assimetria e curtose), considerando os valores contidos entre $-2 /+2$ (para assimetria) e $-7 /+7$ (para curtose) para uma distribuição normal. Este teste de análise da assimetria e curtose realizou-se como forma de apoio e confirmação de uma distribuição normal. De seguida, realizou-se o cálculo das correlações de Pearson entre os estados de humor e a SOT assumindo a significância igual ou inferior a .05.

De forma a dar resposta ao primeiro objetivo do estudo, realizou-se uma análise de regressão linear simples, tomando em consideração os estados de humor como variáveis independentes e a SOT como variável dependente. Esta análise foi efetuada com o suporte de dois testes de multicolinearidade, nomeadamente o teste de Tolerância, que mede o grau em que uma variável explicativa é explicada por todas as outras variáveis explicativas que compõem o modelo) e o Variance Inflation Factor (VIF $=1 /$ Tolerância), sendo que o modelo tem problemas de multicolinearidade quando o teste de Tolerância for menor que 0,1 e se o VIF for superior a 10. Considerou-se para a análise os valores de beta estandardizados e o valor de p, assumindo a significância igual ou inferior a .05 .

Para dar resposta ao segundo objetivo, utilizou-se o tteste para amostras independentes em função do nível de competitividade (i.e., regional vs. nacional) e em função da idade (i.e., 13-15 anos vs. 16-17 anos), considerando como significativo um valor de $p \leq 0,05$. $\mathrm{O}$ efeito da magnitude será calculado de acordo com 


\section{Humor e sobretreinamento em jovens atletas}

as recomendações de Cohen (1988), considerando os seguintes valores de corte relativamente ao efeito: .00 - .19 trivial; .20 - .49 pequeno; .50 - .79 médio; e $>.80$ grande.

\section{RESULTADOS}

Os estados de humor vigor e confusão (positivo) apresentaram as médias mais elevadas, enquanto o estado de humor desajuste ao treino apresentou a média mais baixa. De forma geral, os estados de humor positivos apresentam médias mais elevadas comparativamente aos estados de humor negativos. De acordo com os resultados, os dados apresentam uma distribuição normal dado os valores da assimetria e curtose estarem contidos dentro dos valores de corte previamente descritos. Assim, estes resultados reforçam que os dados apresentam uma distribuição normal.

Os estados de humor de perfil positivo, tais com vigor e confusão (Positivo), demonstraram correlações negativas e significativas com a SOT $(\mathrm{p}>.01)$. Por outro lado, estado de humor negativos (fadiga, hostilidade, depressão, desajuste ao treino, confusão (negativo) e tensão) demonstraram correlações positivas e significativas com a SOT $(p>01)$. A depressão apresenta a maior correlação com a SOT ( $\mathrm{r}$ $=.60, \mathrm{p}>.01)$. Para mais informações ver Tabela 1 .

Tabela 1. Estatísticas descritivas e correlações com a SOT

\begin{tabular}{cccccc}
\hline Variáveis & Média & DP & A & C & Correlação com a SOT \\
\hline Fadiga & 1.04 & .65 & 0.48 & -0.34 & $.23^{* *}$ \\
Vigor & 1.41 & .90 & 0.79 & -0.16 & $-.36^{* *}$ \\
Hostilidade & .93 & .65 & 0.47 & -0.46 & $.31^{* *}$ \\
Depressão & .95 & .67 & 0.62 & 0.59 & $.60^{* *}$ \\
Desajuste ao treino & .83 & .65 & 0.76 & 0.13 & $.47^{* *}$ \\
Confusão (Negativo) & 1.00 & .87 & 0.84 & 0.65 & $.45^{* *}$ \\
Tensão & .93 & .81 & 1.20 & 1.35 & $.15^{* *}$ \\
Confusão (Positivo) & 1.32 & 1.17 & 0.33 & -1.07 & $-.32^{* *}$ \\
SOT & .73 & .29 & -0.40 & -0.51 & 1 \\
\hline
\end{tabular}

Notas: $\mathrm{DP}=$ Desvio Padrão; $\mathrm{A}=$ Assimetria; $\mathrm{C}=$ Curtose; $* * \mathrm{p} \leq .01$

Relativamente às regressões lineares simples, os testes não indicam possíveis problemas de multicolinearidade, dado os valores dos testes de Tolerância e do VIF serem inferiores aos valores de corte. No que diz respeito aos coeficientes do modelo, considerando os estados de Humor como variáveis independentes e a SOT como variável dependente, os estados de humor depressão, desajuste ao treino, confusão (negativo) e tensão apresentaram coeficientes de regressão positivos e significativos. Os estados de humor positivos não apresentam uma associação significativa com a SOT. O modelo apresentou uma variância de $r^{2}=.52(\mathrm{p}<.05)$, ou seja, os estados de humor explicam em $52 \%$ a SOT.

Tabela 2. Regressões lineares simples entre os estados de humor e SOT

\begin{tabular}{lllll}
\hline Variáveis & $\boldsymbol{\beta}$ & $\boldsymbol{p}$ & Tolerância & VIF \\
\hline Fadiga & .07 & .17 & .90 & 1.11 \\
Vigor & .01 & .81 & .67 & 1.49 \\
Hostilidade & .07 & .17 & .81 & 1.23 \\
Depressão & .41 & .00 & .70 & 1.43 \\
Desajuste ao treino & .21 & .00 & .71 & 1.42 \\
Confusão (Negativo) & .23 & .00 & .82 & 1.22 \\
Tensão & .19 & .00 & .93 & 1.08 \\
Confusão (Positivo) & -.07 & .22 & .74 & 1.35 \\
\hline Notas: $\beta=$ coeficiente padronizado; VIF = Variance Inflation Factor
\end{tabular}

Notas: $\beta=$ coeficiente padronizado; VIF = Variance Inflation Factor 


\section{Carvalho et al.}

A Tabela 3 apresenta as comparações dos estados de humor e da SOT em função do nível competitivo. $\mathrm{O}$ teste de Levene não apresentou diferenças significativas $(\mathrm{p}<.05)$ para a igualdade de variâncias. Foram encontradas diferenças estatisticamente significativas no estado de humor hostilidade e depressão em função do nível competitivo. Relativamente à magnitude do efeito, a variável hostilidade apresentou valores de corte triviais e a o estado de humor depressão apresentou valores de corte pequenos. Para mais informações ver Tabela 3.

Tabela 3. T-teste para amostras independentes em função do nível de competitividade

\begin{tabular}{llllll} 
Variáveis & Regional & Nacional & $\mathbf{t}$ & $\boldsymbol{p}$ & EF \\
\hline Fadiga & $.98 \pm .57$ & $1.10 \pm .71$ & -1.40 & .06 & .18 \\
Vigor & $1.45 \pm .90$ & $1.38 \pm .91$ & .57 & .82 & .08 \\
Hostilidade & $.98 \pm .70$ & $.89 \pm .59$ & 1.10 & .00 & .14 \\
Depressão & $.87 \pm .58$ & $1.02 \pm .73$ & -1.68 & .05 & .23 \\
Desajuste ao treino & $.77 \pm .62$ & $.87 \pm .67$ & -1.16 & .71 & .15 \\
Confusão (Negativo) & $1.03 \pm .94$ & $.97 \pm .80$ & .53 & .10 & .07 \\
Tensão & $.98 \pm .85$ & $.88 \pm 0.78$ & .87 & .57 & .12 \\
Confusão (Positivo) & $1.45 \pm 1.21$ & $1.20 \pm 1.12$ & 1.61 & .70 & .21 \\
SOT & $.67 \pm .30$ & $.79 \pm .28$ & -3.02 & .60 & .41 \\
\hline
\end{tabular}

Notas: $p=$ valor de significância; $\mathrm{EF}=$ Magnitude do Efeito.

Na Tabela 4 é apresentado as comparações, desta vez feita entre grupos de idades (13-15 anos vs. 16-17 anos), para os estados de humor e a SOT. O teste de Levene não apresentou diferenças significativas $(\mathrm{p}<.05)$ para a igualdade de variâncias. Foram encontradas diferenças significativas nos estados de humor confusão (positivo) e hostilidade em função da idade. Já na magnitude do efeito, a variável hostilidade apresenta um valor considerado trivial e a variável confusão (positivo) apresenta um valor considerado pequeno.

Tabela 4. T-teste para amostras independentes em função da faixa etária

\begin{tabular}{llllll} 
Variáveis & $\begin{array}{l}\mathbf{1 3 - 1 5} \\
\text { anos }\end{array}$ & $\begin{array}{l}\mathbf{1 6 - 1 7} \\
\text { anos }\end{array}$ & $\mathbf{t}$ & $\boldsymbol{p}$ & $\mathbf{E F}$ \\
\hline Fadiga & $1.08 \pm .62$ & $.96 \pm .71$ & 1.31 & .65 & .18 \\
Vigor & $1.45 \pm .93$ & $1.33 \pm .84$ & .95 & .44 & .13 \\
Hostilidade & $.97 \pm .68$ & $.85 \pm .55$ & 1.28 & .00 & .19 \\
Depressão & $.98 \pm .67$ & $.87 \pm .65$ & 1.18 & .25 & .17 \\
Desajuste ao treino & $.78 \pm .61$ & $.93 \pm .72$ & -1.48 & .25 & .22 \\
Confusão (Negativo) & $1.03 \pm .88$ & $.93 \pm .83$ & .78 & .96 & .12 \\
Tensão & $.97 \pm .85$ & $.82 \pm .72$ & 1.27 & .80 & .19 \\
Confusão (Positivo) & $1.45 \pm 1.22$ & $.99 \pm .96$ & 2.69 & .00 & .42 \\
SOT & $.71 \pm .29$ & $.77 \pm .29$ & -1.41 & .99 & .21 \\
\hline
\end{tabular}

Notas: $p=$ valor de significância; $\mathrm{EF}=$ Magnitude do Efeito. 


\section{Humor e sobretreinamento em jovens atletas}

\section{DISCUSSÃO}

Este estudo teve como objetivo principal analisar a relação entre os estados de humor e a SOT. Em segundo lugar, procurou-se analisar as possíveis diferenças nos estados de humor e na SOT em função do nível competitivo e em função das idades. Os resultados demonstram que existe uma relação significativa entre os estados de humor negativos e a SOT, estando os estados de humor de perfil negativo, como a depressão, o desajuste ao treino, a confusão (negativo) e a tensão positivamente relacionados com a SOT. Relativamente ao segundo objetivo, foram encontradas diferenças estatisticamente significativas nos estados de humor hostilidade e depressão em função do nível competitivo. No que diz respeito à comparação de grupos em função da idade, foram encontradas diferenças significativas nos estados de humor confusão (positivo) e hostilidade. A magnitude do efeito variou entre valores triviais e pequenos.

\section{A relação entre os estados de humor e a SOT}

De acordo com os resultados, os estados de humor negativos depressão, desajuste ao treino, confusão (negativo) e tensão mostraram uma associação positiva e significativa com a SOT. Estes dados corroboram com os achados de outros estudos (e.g., Meeusen et al., 2013) em que estados de humor de perfil negativo aparecem significativamente associados com a SOT. Assim, quanto maior forem os valores de sensação de depressão, maiores perceções de desajuste do treino, de confusão relativamente ao treino e sensação de tensão, maiores serão os valores de SOT apresentados por jovens atletas. Estes dados apresentam um acréscimo na literatura na medida em que os estados de humor negativos podem ser responsáveis pela SOT em atletas jovens Portugueses. De facto, este estudo é o primeiro a analisar a relação entre os estados de humor e a SOT em jovens atletas, sendo que os anteriores apenas consideraram atletas adultos (e.g., Cadegiani, \& Kater, 2018). Acrescido, este é o primeiro estudo a examinar as associações propostas em atletas portugueses. As evidências são congruentes com a literatura, em que estados de humor negativos, especificamente, depressão, desajuste ao treino, confusão (negativo) e tensão apresentaram uma associação positiva e significativa com a SOT.

Pelo contrário, os nossos resultados indicam que os estados de humor positivos como o vigor a confusão (positivo) não apresentaram uma associação significativa com a SOT. Estes resultados não suportam a literatura existente (Schmikli et al., 2011) onde os estados de humor positivos evidenciam uma associação negativa e significativa com a SOT. Estes resultados poderão estar relacionados com a idade dos atletas. Atletas mais jovens poderão ter alguma dificuldade em caracterizar estados de humor positivos devido a incapacidade de lidar (coping) com o stress desportivo imposto em idades jovens (Aoki et al. 2017) causado por fatores externos como pressão dos pais e treinadores e até mesmo problemas pessoais (Grant et al., 2012). Inclusive, a literatura (Mitic et al., 2020) menciona que atletas com idades inferiores a 17 anos apresentam uma associação positiva entre a SOT e a pressão dos pais e treinadores, isto quando em comparação com atletas com idades superiores a 17 anos. Para além disso, Gustafsson et al. (2008) menciona que, estados de humor positivos (e.g. Vigor), são pouco caracterizados na SOT quando em comparação a estados de humor negativos (e.g. Fadiga), ou seja, vários atletas apresentaram associação positiva e significativa entre estados de humor negativos e a SOT, porém, sem apresentar associação significativa entre estados de humor positivos (e.g. Vigor) e a SOT. Nesse sentido, é necessário ter em consideração que, quando em lidar com atletas jovens, a existência de estados de humor positivos (e.g. Vigor) que não sejam significativamente reduzidos, não implicam necessariamente uma inexistência de SOT, sendo por isso necessário ter em consideração todos os estados de humor.

\section{Estados de humor e SOT em função do nível competitivo}

Foram encontradas diferenças significativas no estado de humor hostilidade e depressão em função do nível competitivo, sendo os valores mais elevados para a depressão no nível competitivo nacional e valores mais elevados para a hostilidade no nível competitivo regional. Os valores mais elevados para a depressão no nível nacional corroboram com a literatura que menciona que níveis competitivos mais elevados apresentam valores mais elevados de estados de humor negativos, como hostilidade e depressão (Andrade et al., 2019). Estes dados indicam que quanto mais elevado for o nível competitivo mais elevado serão os sintomas associados à depressão, possivelmente, devido ao desajuste da carga de treino aplicado nestes atletas (Matos et al., 2011) e à elevada 


\section{Carvalho et al.}

carga horária (Lastella et al., 2014). Estes resultados sugerem a necessidade de monitorização de estados de humor e a importância do respeito pelo descanso e uma carga horária mais ajustada (Carfagno et al., 2014), aspetos esses, controlados, maioritariamente, pelo treinador e até mesmo pelos pais dos atletas, que, ao desrespeitarem essas necessidades, provocam, muitas vezes, o impedimento do atleta chegar a profissional (Matos, 2011).

Porém, o mesmo não acontece relativamente à hostilidade que, de acordo com os nossos resultados, apresenta-se mais elevada no nível competitivo regional. Estes resultados são ligeiramente diferentes dos existentes na literatura que menciona a existência de diferenças significativas nos estados de humor hostilidade e depressão, relativamente ao nível que os atletas competem (Andrade et al., 2019) e que níveis competitivos mais elevados apresentam estados de humor de perfil negativo, como hostilidade mais evidentes (O’Connor, 2007). Porém, Boldizsár et al. (2016) menciona que, apesar de níveis competitivos mais elevados, habitualmente, apresentarem valores mais elevados de hostilidade, um nível de sucesso mais reduzido também apresenta associações com o estado de humor hostilidade, justificando, dessa forma, que atletas que se encontrem num nível mais baixo (e.g., regional) podem apresentar valores médios mais elevados de hostilidade. Para além disso, atletas que possuem habilidades mais elevadas e que se encontrem em níveis competitivos mais elevados têm também uma maior capacidade de gestão de hostilidade, pois o nível de experiência competitiva permite que esses mesmos atletas consigam lidar (coping) com esse estado de humor, isto em comparação com atletas que se encontrem em níveis competitivos mais baixos (Sofia et al., 2017). Estas evidências demonstram a necessidade, num nível competitivo mais baixo, de existir tarefas que promovam o sucesso, de forma a aumentar a confiança dos atletas, estando a mesma associada a níveis mais reduzidos de hostilidade, e a necessidade de aplicação de estratégias de coping permitindo que os atletas possam lidar com esse estado de humor (Mowlaie et al., 2011).

\section{Estados de humor e SOT em função da idade}

Foram encontradas diferenças significativas nos estados de humor hostilidade e confusão (positivo) em função da idade, sendo que o grupo com idades compreendias entre os 13-15 anos apresentou os valores mais elevados. Os restantes estados de humor, bem como a SOT, não apresentaram diferenças significativas em função da idade.

Existe uma concordância com a literatura relativamente ao estado de humor hostilidade, que se apresenta mais elevado em idades mais reduzidas (Aoki et al., 2017). Ou seja, existe uma tendência para redução de valores de estados de humor negativos com o avançar da idade, isto pois em idades mais reduzidas existe um desenvolvimento inadequado de capacidade de resolução de problemas causando dessa forma valores mais elevados de hostilidade (Lotfian et al., 2011). Desta forma, é necessário promover e facilitar recursos que permitam a atletas, nestas idades, responderem a situações desportivas que provoquem conflitos e situações de hostilidade (Muñoz-Villena et al., 2020). Porém, apesar da concordância relativamente ao estado de humor negativo, existe uma discordância, relativamente ao estado de humor Confusão (positivo). O motivo destes resultados, relativamente à idade, poderá ser especulado, devido às comparações realizadas na literatura serem habitualmente em idades mais avançadas (Aoki et al., 2017) ou entre idades adultas e jovens, sendo que idades mais avançadas tendem a demonstrar nestados de humor positivos mais elevados (Reynoso-Sánchez et al., 2021), mostrando dessa forma então a necessidade de maior investigação dos estados de humor e da SOT em idades mais jovens (Armstrong et al., 2011). Para além disso, a literatura (Phillips, 2015) define estágios de desenvolvimento de carreira por grupos de idades, sendo que idades até aos 14 anos encontram-se no estágio de crescimento, ou seja, uma fase de aprendizagem onde os indivíduos encontramse a tentar perceber quais os seus gostos e as suas vocações, trata-se de uma fase de experiências. A partir dessa idade até aos 24 entra o estágio de exploração onde é necessário o indivíduo começar a destacar-se e a perseguir uma área vocacional mais focada. As responsabilidades são maiores, assim como o risco para o insucesso. Estando os atletas de idades compreendidas entre os 16-17 anos, num estágio onde a probabilidade para insucesso é maior e onde existe pressão dos pais e treinadores para o sucesso desportivo (Rice et al., 2016), os valores médios de estados de humor positivos poderão ser mais reduzidos em comparação com o grupo mais jovem (13-15 anos) que se encontra numa fase mais lúdica (Chennaoui et al., 2016). Existe, neste grupo de idades, a necessidade de experiências de divertimento, 


\section{Humor e sobretreinamento em jovens atletas}

que fomentem o espírito em equipa e o envolvimento com outras pessoas de forma a tornar a atividade mais prazerosa, pois o divertimento e o prazer na execução da atividade têm sido associados a estados de humor positivos (McCarthy, 2011).

\section{Limitações}

Este estudo apresenta fortes concordâncias com a literatura preenchendo algumas lacunas da mesma como por exemplo os valores reduzidos de amostra. Porém é necessário perceber que a natureza transversal do mesmo impossibilita de analisar o efeito que os estados de humor possam ter na SOT. Para além disso a amostra analisada era heterogénea, o que impossibilitou a comparação entre diferentes modalidades e entre género. A faixa etária também se mostrou muito contida entre os 13 e os 17 anos de idades o que não permitiu realizar esta análise em atletas de outras idades. Assim, estudos futuros poderão ser de natureza longitudinal de forma a obter resultados de causalidade e, também, englobar outras variáveis como diferentes modalidades, realizando comparações entre desportos coletivos e individuais, comparações entre géneros e uma faixa etária mais alargada.

É de referir que este estudo é o primeiro, de acordo com a nossa pesquisa bibliográfica, a considerar os estados de humor positivos e negativos como determinantes da SOT em jovens atletas portugueses, sendo por isso, os resultados confinados para amostras semelhantes. Estudos futuros poderão considerar estas ligações com outras amostras de forma a criar um padrão de generalização dos resultados de forma que se criem intervenções eficientes na redução da SOT em atletas do nível competitivo mais alto ou idades mais avançadas.

\section{CONCLUSÕES}

Este estudo indica que existe uma associação entre os estados de humor negativos, especificamente os estados de humor como a depressão, o desajuste ao treino, a confusão (negativo) e a tensão positivamente relacionados com a SOT. Os resultados deste estudo demonstram, também, diferenças significativas no estado de humor hostilidade e depressão em função do nível competitivo. Por último, foram encontradas diferenças significativas nos estados de humor hostilidade e confusão (positivo) em função da idade. Estes resultados mostram que é necessário um diferente tipo de monotorização para cada tipo de atleta tendo em consideração aspetos como a idade e o nível competitivo. Acrescido, este estudo evidencia a praticabilidade na análise dos estados de humor em jovens atletas sendo que estados de humor negativos podem estar positivamente associados à SOT.

\section{IMPLICAÇÕES PRÁCTICAS}

A análise dos estados de humor em jovens atletas permite a existência de uma prevenção perante a SOT. É importante perceber o impacto que treinadores, clubes e pais (Merkel, 2013) têm na prevenção da SOT. Essa prevenção ocorre através do tipo de interação do treinador para com o atleta, quando este utiliza uma abordagem mais humanista e uma comunicação que abrange não só o tópico desportivo como outros tópicos, que permitam ao atleta um desenvolvimento social e psicológico dentro da prática desportiva (Mitic et al., 2020). Para além disso, é necessário existir uma comunicação efetiva não só entre o treinador e o atleta como também entre o treinador e os pais de forma a estar a par da carga horária existente na vida do atleta tendo em conta a atividade escolar e outro tipo de atividades extracurriculares mantendo assim um equilíbrio entre o descanso e atividade física e permitindo, também, a existência de vida social na rotina do atleta (Matos et al., 2011). Este tipo de abordagens permite um aumento de estados de humor positivos e uma redução de estados de humor negativos evitando dessa forma a SOT (Rice et al., 2016).

Outras variáveis são importantes ter em conta como o tipo de modalidade e o género do atleta, sendo que modalidades individuais apresentam valores mais elevados de SOT (Purcell et al., 2019) e de estados de humor negativos (Nixdorf et al., 2016), e o género feminino é mais propício para o aparecimento de SOT (Matos et al., 2011) e com tendência para estados de humor negativos mais elevados (Rice et al., 2016).

\section{REFERÊNCIAS}

1. Andrade, A., Bevilacqua, G., Casagrande, P., Brandt, R., \& Coimbra, D. (2019). Sleep quality associated with mood in elite athletes. The Physician and Sportsmedicine, 47(3), 312-317. https://doi.org/10.1080/00913847.2018.1553467

2. Aoki, M., Arruda, A., Freitas, C., Miloski, B., Marcelino, P., Drago, G., \& Moreira, A. (2017). Monitoring training loads, mood states, and jump 


\section{Carvalho et al.}

performance over two periodized training mesocycles in elite young volleyball players. International Journal of Sports Science \& Coaching, 12(1):

130-137. https://doi.org/10.1177/1747954116684394

3. Armstrong, N., \& McManus, A. M. (2011). The Elite Young Athlete. Medicine of Sports Science, 56, 97-105. https://doi.org/10.1159/000320636

4. Bell, L., Ruddock, A., Maden-Wilkinson, T., \& Rogerson, D. (2020). Overreaching and overtraining in strength sports and resistance training: A scoping review. Journal of Sports Sciences, 38(16), 1897-1912. https://doi.org/10.1080/02640414.2020.1763077

5. Boldizsár, D., Soós, I., Whyte, I., \& Hamar, P. (2016). An investigation into the relationship between pre-competition mood states, age, gender and a national ranking in artistic gymnastics. Journal of human kinetics, 51, 243$252 . \quad$ https://doi.org/10.1515/hukin-20150188Brooks

6. K., \& Carter, J. (2013). Overtraining, exercise, and adrenal insufficiency. Journal of novel physiotherapies, $3(125)$, 11717. https://doi.org/10.4172/2165-7025.1000125

7. Cadegiani, F. (2020). Practical Approach to the Athlete Suspected or at High Risk for OTS. In Overtraining Syndrome in Athletes, Springer 189-223. https://doi.org/10.1007/978-3-03052628-3 11

8. Cadegiani, F., \& Kater, C. (2018). Body composition, metabolism, sleep, psychological and eating patterns of overtraining syndrome: results of the EROS study (EROSPROFILE). Journal of Sports Sciences, 36(16), 1902-1910. https://doi.org/10.1080/02640414.2018.1424498

9. Carfagno, D., \& Hendrix, J. (2014). Overtraining syndrome in the athlete: current clinical practice. Current Sports Medicine Reports, 13(1), 45-51. https://doi.org/10.1249/JSR.0000000000000027

10. Carter, J., Potter, A., \& Brooks, K. (2014). Overtraining syndrome: Causes, consequences, and methods for prevention. Journal of Sport and Human Performance; 2(1), 1-14. https://doi.org/10.12922/jshp.0031.2014
11. Chennaoui, M., Bougard, C., Drogou, C., Langrume, C., Miller, C., Gomez-Merino, D., \& Vergnoux, F. (2016). Stress biomarkers, mood states, and sleep during a major competition: "Success" and "failure" athlete's profile of highlevel swimmers. Frontiers in Physiology, 7, 94. https://doi.org/10.3389/fphys.2016.00094

12. Fernandes, J., Nogueira, R., Andrade, F., Freitas, D. S., Bara Filho, M. (2008). Tradução e adaptação do questionário de sintomas clínicos do Overtraining. Coleção Pesquisa em Educação Física, 7(1), 335-340. https://doi.org/10.1590/19800037.2009v11n $4 \mathrm{p} 457$

13. Grant, C., Van Rensburg, D., Collins, R., Wood, P., \& Du Toit, P. (2012). The Profile of Mood State (POMS) questionnaire as an indicator of Overtraining Syndrome (OTS) in endurance athletes. African Journal for Physical Health Education, Recreation and Dance, 18(1), 23-32. https://doi.org/10520/EJC119823

14. Grandou, C., Wallace, L., Coutts, A., Bell, L., \& Impellizzeri, F. (2020). Symptoms of overtraining in resistance exercise: international crosssectional survey. International Journal of Sports Physiology and Performance, 1, 1-10. https://doi.org/10.1123/ijspp.2019-0825

15. Gustafsson, H., Holmberg, H. C., \& Hassmen, P. (2008). An elite endurance athlete's recovery from underperformance aided by a multidisciplinary sport science support team. European Journal of Sport Science, 8(5), 267-276. https://doi.org/10.1080/17461390802195652

16. Han, C.S.Y., Parsons-Smith, L.R., Terry, P.C. (2020). Mood Profiling in Singapore: CrossCultural Validation and Potential Applications of Mood Profile Clusters. Frontiers in Psychology, 11:665.

https://doi.org/10.3389/fpsyg.2021.624119

17. Hernández Mendo, A. (2011). Biofeedback electromiográfico en la rehabilitación de lesiones de rodilla. Estudio de dos casos en futbolistas profesionales. Cuadernos de Psicología del Deporte, 11(2), 71-80.

18. Hernández Mendo, A. \& Ramos, R. (1996). Informatización del Profile of Mood States de 


\section{Humor e sobretreinamento em jovens atletas}

Mcnair, Lorr y Dropleman (1971). Aplicaciones. En Hernández Mendo y R. Ramos, Introducción a la informática aplicada a la psicología del deporte. Herramientas informáti-cas de uso en las ciencias del deporte (pp.55-85). Madrid: Editorial Ra-Ma.

19. Hernández Mendo, A. (2002)._Actividad física y emoción, a veces felicidad. En P. Fernández Berrocal y N. Ramos Díaz, Corazones Inteligentes (pp.181-194). Barcelona: Kairós.

20. Hernández Mendo, A. \& Ramos, R. (1995). Informatización del Profile of Moode States de McNair, Lorr y Dropleman (1971). Aplicaciones. Revista de Psicología del Deporte, 7-8, 31-50.

21. Kline, R. (2016). Principles and practice of structural equation modelling ( $3^{\text {rd }}$ ed.). New York, The Guildford Press. ISBN: 9781462523344.

22. Kreher, J. (2016). Diagnosis and prevention of overtraining syndrome: an opinion on education strategies. Journal of Sports Medicine, 7, 115. https://doi.org/10.2147/OAJSM.S91657

23. Kreher, J., \& Schwartz, J. (2012). Overtraining syndrome: a practical guide. Sports Health, 4(2), 128-138. https://doi.org/10.1177/1941738111434406

24. Lastella, M., Lovell, G. P., \& Sargent, C. (2014). Athletes' precompetitive sleep behaviour and its relationship with subsequent precompetitive mood and performance. European Journal of Sport Science, 14(1), S123-S130. https://doi.org/10.1080/17461391.2012.660505

25. Lotfian, S., Ziaee, V., Amini, H., \& Mansournia, M. A. (2011). An analysis of anger in adolescent girls who practice the martial arts. International Journal of Pediatrics, 2011. https://doi.org/10.1155/2011/630604

26. Matos, N., Winsley, R., \& Williams, C. A. (2011). Prevalence of nonfunctional overreaching/overtraining in young English athletes. Medicine and Science in Sports and Exercise, $\quad 43(7)$, 1287-1294. https://doi.org/10.1249/MSS.0b013e318207f87b

27. McCarthy, P. J. (2011). Positive emotion in sport performance: current status and future directions. International Review of Sport and Exercise Psychology, 4(1), 50-69. https://doi.org/10.1080/1750984X.2011.560955
28. Meeusen, R., Duclos, M., Foster, C., Fry, A., Gleeson, M., Nieman, D., Raglin, J., Rietjens, G., Steinacker, J., \& Urhausen, A. (2013). Prevention, diagnosis, and treatment of the overtraining syndrome: joint consensus statement of the European College of Sport science and the American College of sports medicine. European Journal of Sports Science, 45, 1-24. https://doi.org/10.1080/17461391.2012.730061

29. Merkel, D. (2013). Youth sport: Positive and negative impact on young athletes. The Journal of Sports Medicine, 4, 151-160. https://doi.org/10.2147/OAJSM.S33556

30. Mitic, P., Stojiljković, N., \& Arsić, I. (2020). Overtraining in student-athletes: do coaches and parents "push too hard?". Facta Universitatis, Series: Teaching, Learning and Teacher Education, 4(1), 027-033. https://doi.org/10.22190/FUTLTE2001027M

31. Mowlaie, M., Besharat, M. A., Pourbohlool, S., \& Azizi, L. (2011). The mediation effects of selfconfidence and sport self-efficacy on the relationship between dimensions of anger and anger control with sport performance. ProcediaSocial and Behavioral Sciences, 30, 138-142. https://doi.org/10.1016/j.sbspro.2011.10.027

32. Muñoz-Villena, A. J., Gómez-López, M., \& González-Hernández, J. (2020). Perfectionism profiles and anger responses: The relevant role of self-esteem in athletes of professional quarries. International journal of environmental research and public health, 17(4), 1416. https://doi.org/10.3390/ijerph17041416

33. Nettle, D., \& Bateson, M. (2012). The evolutionary origins of mood and its disorders. Current Biology, 22(17), 712-721. https://doi.org/10.1016/j.cub.2012.06.020

34. Nixdorf, I., Frank, R., Hautzinger, M., \& Beckmann, J. (2013). Prevalence of depressive symptoms and correlating variables among German elite athletes. Journal of Clinical Sport Psychology, 7(4), 313-326. https://doi.org/10.1123/jcsp.7.4.313

35. Nixdorf, I., Frank, R., \& Beckmann, J. (2016). Comparison of athletes' proneness to depressive symptoms in individual and team sports: Research on psychological mediators in junior elite 


\section{Carvalho et al.}

athletes. Frontiers in Psychology, 7, 893. https://doi.org/10.3389/fpsyg.2016.00893

36. O'Connor, P. (2007). Monitoring and titrating symptoms. Sports Medicine, 37(4), 408-411. https://doi.org/10.2165/00007256-20073704000035

37. Oliveira, B., Cavalcanti Matos, I., Maranhão Neto, G., Rodrigues, F., Monteiro, D., Lattari, E., \& Machado, S. (2021). Una intervención de 16 semanas sobre el estado de ánimo en ancianos: prueba de dos programas de ejercicio: probar dos programas de ejercicio. Cuadernos de Psicología del Deporte, 21(2), 24-31. https://doi.org/10.6018/cpd.433571

38. Phillips, S. D. (2015). Lifespan career development. In P. J. Hartung, M. L. Savickas, \& W. B. Walsh (Eds.), APA handbooks in psychology. APA handbook of career intervention, 1, 99-113. American Psychological Association. https://doi.org/10.1037/14438-006

39. Purcell, R., Gwyther, K., \& Rice, S. (2019). Mental health in elite athletes: increased awareness requires an early intervention framework to respond to athlete needs. Sports Medicine - $\quad$ Open, 5(1), 1-8. https://doi.org/10.1186/s40798-019-0220-1

40. Reynoso-Sánchez, L., Pérez-Verduzco, G., Celestino-Sánchez, M., López-Walle, J., Zamarripa, J., Rangel-Colmenero, B., MuñozHelú, H., \& Hernández-Cruz, G. (2021). Competitive Recovery-Stress and Mood States in Mexican Youth Athletes. Frontiers in Psychology, 11, 627828 . https://doi.org/10.3389/fpsyg.2020.627828

41. Rice, S. M., Purcell, R., De Silva, S., Mawren, D., McGorry, P., \& Parker, A. (2016). The Mental Health of Elite Athletes: A Narrative Systematic Review. Sports Medicine, 46(9), 1333-1353. https://doi.org/10.1007/s40279-016-0492-2
42. Roberts, R.J., \& Lane, A.M. (2021). Mood Responses and Regulation Strategies Used During COVID-19 Among Boxers and Coaches. Frontiers in Psychology, 12: 624119. https://doi.org/10.3389/fpsyg.2021.624119

43. Schmikli, S., Brink, M., De Vries, W., \& Backx, F. (2011). Can we detect non-functional overreaching in young elite soccer players and middle-long distance runners using field performance tests? British Journal of Sports Medicine, 45(8), 631-636. https://doi.org/10.1136/bjsm.2009.067462

44. Sofia, R., \& Cruz, J. F. A. (2017). Unveiling anger and aggression in sports: The effects of type of sport, competitive category and success level. Revista de psicología del deporte, 26(2), 21-28. ISSN 1988-5636

45. Ten Haaf, T., van Staveren, S., Oudenhoven, E., Piacentini, M., Meeusen, R., Roelands, B., Koenderman, L., Daanen, H., Foster, C., \& De Koning, J. (2017). Prediction of functional overreaching from subjective fatigue and readiness to train after only 3 days of cycling. International Journal of Sports Physiology and Performance, 12(2), 82-87. https://doi.org/10.1123/ijspp.2016-0404

46. Tobar, D. (2012). Trait anxiety and mood state responses to overtraining in men and women college swimmers. International Journal of Sport and Exercise Psychology, 10(2), 135-148. https://doi.org/10.1080/1612197X.2012.666399

47. Viana, M., Almeida, P., \& Santos, R.(2001). Adaptação portuguesa da versão reduzida do Perfil de Estados de Humor-POMS. Análise Psicológica, 19(1), 77-92. https://doi.org/10.14417/ap.345

48. Yoshihara, K., Hiramoto, T., Sudo, N., \& Kubo, C. (2011). Profile of mood states and stress-related biochemical indices in long-term yoga practitioners. BioPsychoSocial Medicine, 5(1), 6. https://doi.org/10.1186/1751-0759-5-6 